\title{
Stabilization of a one-dimensional short-range model atom in intense laser fields
}

\author{
Q Su†, B P Irving †, C W Johnson† and J H Eberly $\ddagger$ \\ $\dagger$ Department of Physics, Illinois State University, Normal, IL 61790, USA \\ $\ddagger$ Department of Physics and Astronomy, University of Rochester, Rochester, NY 14627, USA
}

Received 18 July 1996

\begin{abstract}
The stabilization of atoms in the presence of very intense and short laser pulses has been proposed and confirmed in various numerical simulations (e.g. Su $e t a l$ ). A question was then raised by several authors (Chen and Bernstein, Krainov and Preobrazhenskii, and Geltman) concerning the possibility of producing such stabilization in a one-dimensional model atom with zero range of electron-nucleus interaction. In this paper we report evidence of stabilization through the suppression of ionization for such a model atom. We find that the use of a highfrequency laser pulse is necessary for such a realization.
\end{abstract}

\section{Introduction}

The ionization of an atomic system in the presence of a laser field at the $10^{13} \mathrm{~W} \mathrm{~cm}^{-2}$ intensity level is typically very poorly predicted by any perturbative formula. For such laser intensities one even has to be concerned with whether the ionization grows in time with a unique rate. Theoretical investigations of such fundamental questions have partially resorted to numerical solutions of the time-dependent Schrödinger equation (Kulander 1987, Javanainen et al 1988, Kulander et al 1991, Su 1993, Burnett et al 1993). Such time-dependent analyses revealed signatures of the ionization that confirmed, at least qualitatively, agreement with observations obtained experimentally. This includes the above threshold ionization (ATI) electron energy spectrum (Agostini et al 1979, Eberly et al 1991, Bucksbaum and Freeman 1991) and the generation of high-order harmonics in very intense laser pulses (McPherson et al 1987, Ferray et al 1988, Balcou 1996).

In addition, predictions of so-called atomic stabilization, or laser stabilization of atoms, in very intense laser light have appeared (e.g. Gersten and Mittleman 1976, Gavrila and Kaminski 1984). Adiabatic stabilization is the counter-intuitive steady-state situation in which ionization of an atomic electron is prevented under the influence of an asymptotically high-frequency and high-intensity radiation field of constant amplitude. Adiabatic stabilization is usually associated with specific dressed-field eigenstates in the Kramers-Henneberger picture (see Pont et al 1988, Kramers 1956, Henneberger 1968). Dynamic stabilization is similar but not a steady-state phenomenon. It refers to the strong suppression of ionization initiated during a rapidly turned-on laser pulse. Adiabatic stabilization is generally presumed to be a limiting version of dynamic stabilization, and which can be achieved if the laser pulse can be turned on sufficiently slowly and to a very high intensity, without suffering $100 \%$ ionization in the process. It has never been shown rigorously that this is possible for any finite pulse-switching, although it is strongly suggested 
by many numerical studies. Rigorous analysis of dynamic stabilization has recently been initiated (see Fring et al 1996). Dynamic stabilization was first observed for a realistically pulsed laser interaction on a one-dimensional model atom with a long-range binding potential (Su et al 1990, Su and Eberly 1990, Su 1993). Stabilization has also been confirmed in calculations for a three-dimensional hydrogen atom (Dörr et al 1990, Pont and Gavrila 1990, Dörr et al 1991, Kulander et al 1991). Both kinds of stabilization are described in the recent review by Eberly and Kulander (1993).

However, in a recent study of the photo-detachment of a model negative ion, Geltman has come to conclusions very different from these authors (Geltman 1994, 1995). In Geltman's study the electron-nucleus interaction has the form of a delta function $V(x)=$ $-B \delta(x)$. Previous delta-potential studies also exist (Grozdanov et al 1990, Mostowski and Eberly 1991, Sanpera et al 1993, Volkova et al 1994). On the basis of his results, Geltman questioned strongly the existence of both adiabatic and dynamic stabilization. He discussed the ionization of his one-dimensional system in three regions, i.e. the regions of multiphoton ionization (MPI), tunnelling ionization (TI), and over-the-barrier ionization (OBI).

In the present paper, we report the results of our recent time-dependent ionization calculations which also employ a zero-range model negative ion (for stabilization in another model negative ion, see Grobe and Fedorov (1992)). In the following discussions, we will use ionization and photo-detachment interchangeably even though it is more realistically called detachment for a short-range potential. We show convincing evidence of dynamic stabilization for certain conditions of intense laser pulses. Earlier studies of stabilization for such a potential have already been carried out but in a slightly different context. In that case the laser was assumed to be switched on abruptly (Sanpera et al 1993). Such an assumption is not a safe one in maintaining a stabilized state of an atomic electron. The question of whether a bound electron can survive the pulse turn-on has not been addressed for this potential.

We have made our calculations with the electron initially in the sole negative-energy state of the field-free delta potential and exposed to pulses of both smooth and abrupt shapes. We find clear evidence of stabilization as a distinct process in super-strong laser fields in addition to the MPI, TI and OBI processes under other laser conditions. We suggest that such a stabilization process takes place only when the field frequency is high enough that the optical period is considerably shorter than the electron orbital period in the absence of the field. It has already been shown that this frequency condition can be safely violated if the potential is not zero-range in character (Law et al 1991).

\section{The model atom}

To simulate the one-dimensional delta potential we take a one-dimensional square well (with depth $D$ and width $w$, chosen in such a way that $D \times w=B$ ). We then reduce the width $w$ and increase $D=B / w$, in such a way that the total area $B$ is unchanged. In the limit $w \rightarrow 0$ the energy and wavefunction of the only bound state will approach that of a delta potential

$$
V(x)=-B \delta(x)
$$

with the only bound-state energy given by

$$
W_{b}=-\frac{m B^{2}}{2 \hbar^{2}}
$$


and the corresponding eigenfunction

$$
u_{b}(x)=\frac{\sqrt{m B}}{\hbar} \mathrm{e}^{-m B|x| / \hbar^{2}} .
$$

Here $B$ is the strength of the potential, $m$ is the mass of the electron and $\hbar$ is Planck's constant. The convergence of the energy to the delta-potential energy goes approximately as $w \times B^{4}$.

The eigenvalue problem of the field-free system is solved by the standard finite difference method on a space grid. This involves the replacement of the second derivative by the finite difference, three-point formula. The resulting matrix equation is diagonalized by placing the atom in a large box and applying the boundary condition of the first kind. For a typical run the box size is 2000 (we adopt atomic units hereafter unless otherwise specified), and the potential width is chosen at $w=0.244$. With $B=\frac{1}{2}$, the energy comes out to be 0.1245 , about $0.4 \%$ off the exact value. The eigenfunction converges to the analytical form with about the same accuracy.

\section{Ionization in weak fields}

The coupling of the model atom with a laser field occurs through a dipole interaction Hamiltonian. The resulting time-dependent Schrödinger equation has the form

$$
\left(-\frac{1}{2} \frac{\partial^{2}}{\partial x^{2}}+V(x)+x E_{0} f(t) \sin \omega t\right) \Psi(x, t)=\mathrm{i} \frac{\partial}{\partial t} \Psi(x, t) .
$$

This equation will be solved for various laser intensities $\left(I=E_{0}^{2}\right)$ and frequencies $\omega$. Here $f(t) \leqslant 1$ describes the shape of the laser pulse, which we switch on and off smoothly according to $f(t)=\sin ^{2}\left(\pi t / T_{o n}\right)$, for $0 \leqslant t \leqslant T_{o n}$. A plot of such a pulse envelope is shown in figure 1 . Between the pulse turn-on and the turn-off, the field is kept at a constant peak value.

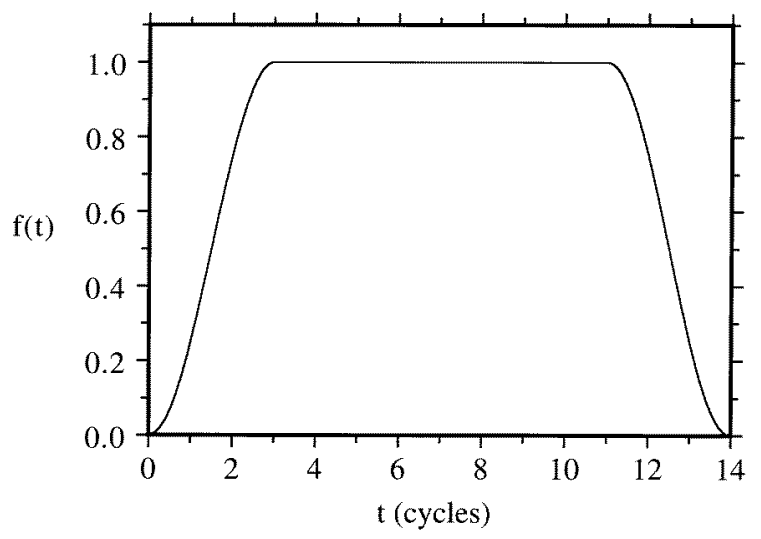

Figure 1. The pulse envelope function of a laser field, showing a smooth turn-on, a constant peak value, followed by a smooth turn-off.

The Schrödinger equation is first transformed into the $p A$ form with the transformation

$$
\Psi(x, t)=\mathrm{e}^{\mathrm{i} A(t) x / c} \Psi_{A}(x, t) .
$$


The resulting equation

$$
\left[-\frac{1}{2}\left(-\mathrm{i} \frac{\partial}{\partial x}+\frac{A(t)}{c}\right)^{2}+V(x)\right] \Psi_{A}(x)=\mathrm{i} \frac{\partial}{\partial t} \Psi_{A}
$$

is then integrated by the so-called split operator method (Feit et al 1982). The one-step wavefunction advancement in time follows

$$
\Psi_{A}(t+\Delta t)=F^{-1}\left[\mathrm{e}^{-\frac{\mathrm{i}}{4}(k+A / c)^{2} \Delta t} F\left[\mathrm{e}^{-\mathrm{i} V \Delta t} F^{-1}\left[\mathrm{e}^{\frac{\mathrm{i}}{4}(k+A / c)^{2} \Delta t} F\left[\Psi_{A}\right]\right]\right]\right] .
$$

Here $F[\ldots]$ and $F^{-1}[\ldots]$ represent the Fourier and the inverse Fourier transforms as defined below:

$$
F[\Psi] \equiv \tilde{\Psi} \equiv \int \frac{\mathrm{d} x}{\sqrt{2 \pi}} \mathrm{e}^{-\mathrm{i} k x} \Psi(x) ; \quad F^{-1}[\tilde{\Psi}] \equiv \int \frac{\mathrm{d} k}{\sqrt{2 \pi}} \mathrm{e}^{\mathrm{i} k x} \tilde{\Psi}(k) .
$$

The resulting wavefunction is then back-transformed from $p A$ to $d E$ form.
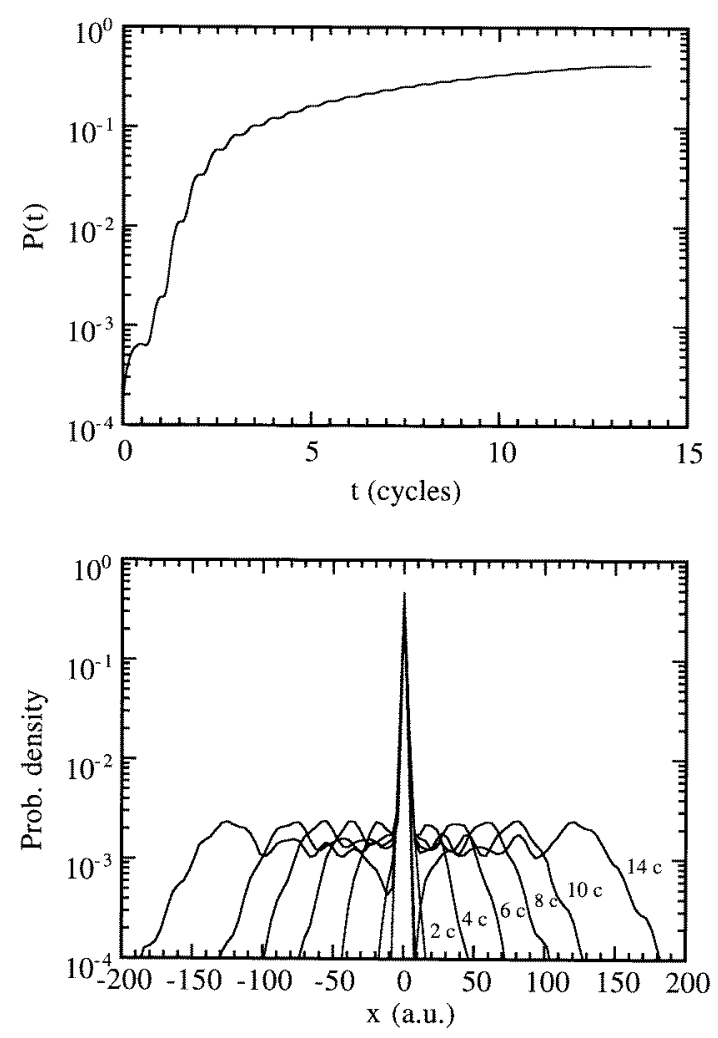

Figure 2. Total ionization probability is plotted (a) as a function of time for a laser with $\omega=$ 0.2 au and $E_{0}=0.01$ au. The pulse turn-on took two optical cycles; the constant peak field lasted 10 cycles; the turn-off took two cycles. In $(b)$, the probability densities are plotted at selected instances $(0,2,4,6,8,10$ and 14 cycles) for the same laser pulse conditions.

We have obtained solutions for $\Psi$ for one-photon ionization with laser-atom parameters suggested by Geltman's (1994) investigation. They are $B=\frac{1}{2}, \omega=0.2, E_{0}=0.1$. Following Geltman, the pulse is turned on in two optical cycles, held at its peak for 10 cycles, and turned off in two cycles. Figure 2(a) shows the total probability of ionization as a function of time, defined as

$$
P(t)=1-\left|\left\langle u_{b} \mid \Psi(t)\right\rangle\right|^{2} .
$$

Some snapshots of the wavepacket during the course of the laser pulse are plotted in figure $2(b)$. These plots correspond to Geltman's results (see figures 2 and 5 of Geltman (1994)). 

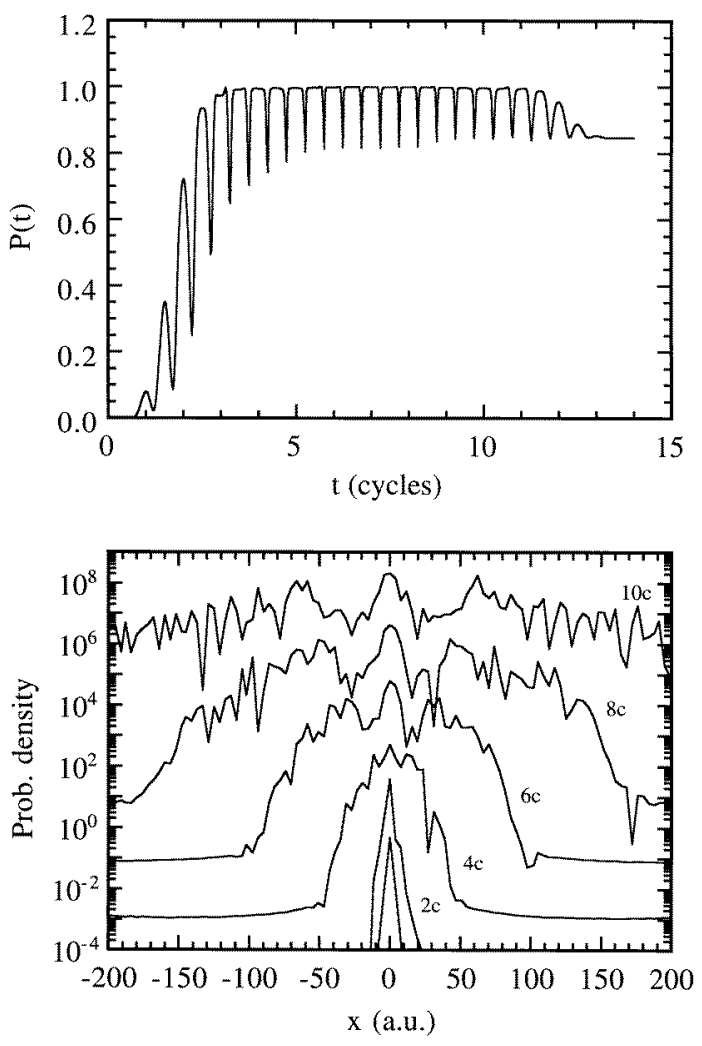

Figure 3. Total ionization probability is plotted (a) as a function of time for a laser with $\omega=1$ and $E_{0}=4 \mathrm{au}$. The sine-squared pulse turn-on took seven optical cycles; the turn-off took seven cycles. Residual population in bound levels after the pulse are visible. It can be attributed to the stabilization of the atom during the pulse. In $(b)$, the probability densities are plotted at selected instances $(0,2,4,6,8$ and 10 cycles) for the same laser pulse conditions. Note that the vertical scale for each plot has been enlarged by two decades for a better view.

\section{Stabilization in super-strong fields}

To find evidence of stabilization in super-strong fields, it is known that it is necessary to go to high laser frequency. Therefore, we recalculated the ionization probability with parameters $B=\frac{1}{2}, \omega=1$, and $E_{0}=4$. The pulse was turned on in seven cycles before being turned off in another seven cycles. The ionization probability as defined in equation (9) is plotted in figure 3(a), which shows oscillations of the projection of the wavefunction onto the field-free energy states. Twice every optical cycle there is a large projection. Such a high frequency oscillation has been discussed in our earlier publications about stabilization (Su et al 1990, Su and Eberly 1990). The oscillation is understood to arise from the large-amplitude quiver motion of a nearly free electron in the presence of the laser field.

This high-intensity excitation also has an effect on the spatial localization of the electron, as shown in figure $3(b)$. The total amount of ionization after the pulse is smoothly turned off at the fourteenth cycle is nearly $85 \%$, i.e. $15 \%$ of the bound-electron probability survives. Snapshots of the electron wavefunction taken during the pulse at selected instances are shown in figure $3(b)$. Here the localization of the electron wavepacket is evident. Such a localized electron wavepacket oscillates about the nucleus at $x=0$ at the laser frequency. As a result, twice each cycle the packet has very little overlap with $u_{b}(x)$, which produces the oscillation in ionization probability at twice the laser frequency shown in figure $3(a)$ and mentioned above. 


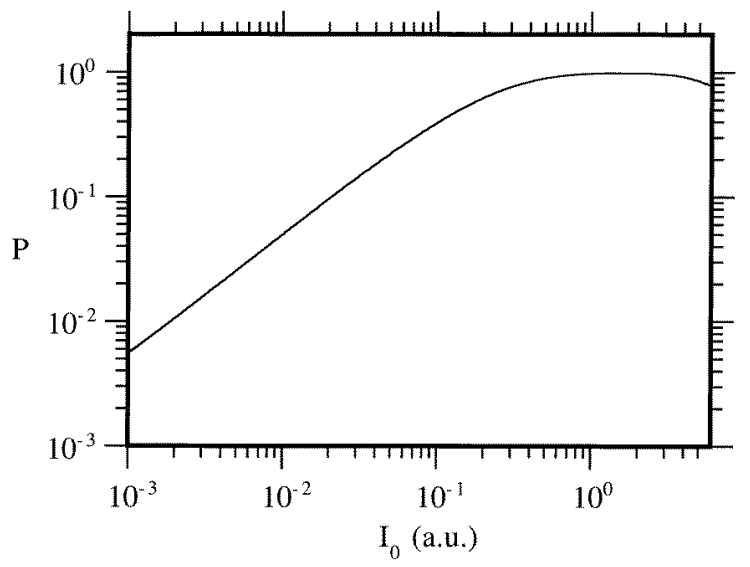

Figure 4. End-of-pulse ionization is plotted as a function of laser peak intensity. The laser pulse turned on and off (sine-squared ramps) in three optical cycles. In between, the constant laser field lasted eight optical cycles. The frequency of the laser field oscillation was $\omega=1$ au. An ionization suppression is visible after $I_{0}=3$ au. (The atomic units of intensity $=3.5 \times 10^{16} \mathrm{~W} \mathrm{~cm}^{-2}$.)

\section{The transition to stabilization and beyond}

We have also made a series of one-photon ionization computations for parameters similar to figure 2 except for the peak intensity value and the pulse shape. The pulse was turned on in three cycles, and held for eight cycles at the peak value before being turned off in another three cycles. The ionization probability after each pulse is plotted as a function of the peak intensity $\left(I_{0}=E_{0}^{2}\right)$ in figure 4 , which shows three characteristic ionization regions (Su and Eberly 1991). The first region has been referred to as the weak-field region. In figure 4 this means the region $I_{0} \ll 0.2$. On the $\log -\log$ scale, in this region the curve approaches a straight line with slope 1 . This is the perturbative prediction for a one-photon process.

The second region is referred to as the strong-field region. In figure 4 it covers a small region around $I_{0} \approx 1$. Here almost $100 \%$ of the population ends up in the continuum after the pulse. The third region is referred to as the super-strong-field region. In figure 4 it corresponds to the region $I_{0}>3$. In this region we find that ionization is more suppressed as the peak intensity is increased, and figure 5 shows that the degree of stabilization at $I_{0}=6.8$ is, for instance, close to $25 \%$.

Figure 5 also shows that the stabilization shown in figure 4 is not monotonic. From $I_{0}=6.8$ to $I_{0}=10.3$ a recovery of ionization appears (Su 1996, Su et al 1996; see also Yao and Chu 1992, Millack 1993). Such a recovery structure is to be expected when an atom stabilizes in super-strong laser fields. At still higher intensity, around $I_{0} \approx 20$ in figure 5, the total ionization probability starts to increase slowly to $100 \%$ again. Such an increase in ionization has been observed in other models, either when the field strength is strong or the pulse turn-on is short (Su et al 1990, Kulander et al 1991), as found in the square pulse case discussed in section 6 . If the rise time is held fixed and the peak intensity is increased, the bandwidth that the bound population experiences will eventually approach the square pulse case. It appears in our example that an intensity increase rate of more than five atomic units per cycle will lead to such an ionization. The mechanism for the small 


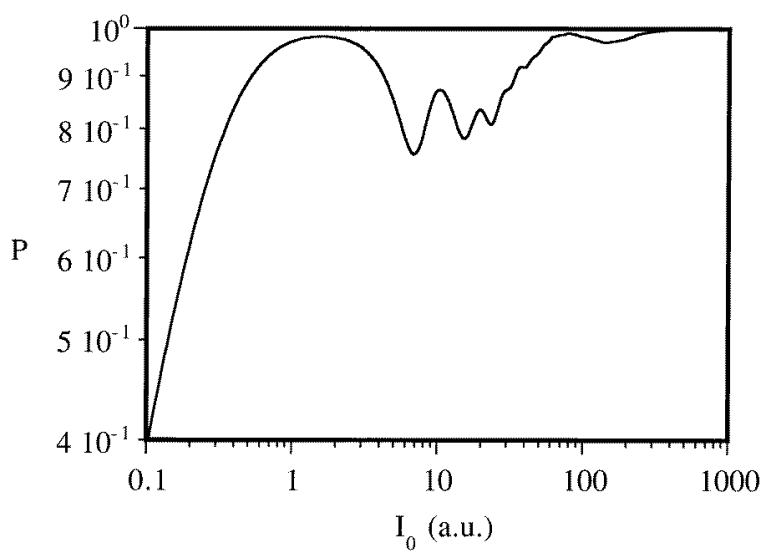

Figure 5. A replot of figure 4 on a wider intensity range, showing recovery structure during the stabilization.

but noticeable oscillations during the stabilization and beyond will be discussed separately in a later paper.

\section{Remarks on the pulse shape}

Lasers have smooth pulse shapes. Such a feature can be taken into account in numerical studies explicitly, but is frequently overlooked. One of the reasons is that most ionization effects do not exhibit differences on a qualitative basis with the selection of different pulse shapes. Therefore, to choose an over-simplified square pulse is simply very convenient. In the strong laser intensity limit, on the other hand, the pulse shape becomes crucially important since ionization can be accomplished quite effectively during the turn-on or turnoff edges of the pulse (e.g. Grobe and Fedorov 1992, Haan 1996). Stabilization is an effect that is definitely pulse-shape sensitive.

To support this remark, in figure 6 we have compared our computed end-of-pulse ionization for a smooth pulse with that of a square pulse. The smooth pulse is the same one used for the calculation shown in figure 5, but re-plotted. The sine-squared turn-on, constant peak value and sine-squared turn-off periods are separately fixed at three, eight and three cycles. The 14-cycle square pulse is turned on and off instantaneously. It is evident from figure 6 that the square pulse ionization curve grows smoothly, reaches $100 \%$ around $I_{0} \approx 0.2$, and stays at $100 \%$ for all greater peak intensities.

The explanation of zero stabilization for the square pulse can be thought of as due to the large frequency band in the original pulse which couples the initial bound state to a large number of continuum states. The result is a burst of ionization during the turn-on cycles. It appears Geltman has arrived at his observations of no stabilization partially from results due to pulses without turn-on (Geltman 1995). It comes without surprise that no stabilization was seen in his results. His other investigations (Geltman 1994, 1995) in very high laser intensities including pulses with turn-on suffered from numerical convergence problems, and technical difficulty has also been indicated (Geltman 1995). Conclusive statements from such a limited set of investigations should be made very cautiously. 


\section{Comments on the high-frequency condition}

We would like to mention that the high-frequency condition makes the so-called over-thebarrier ionization picture a very poor one. In that picture, ionization can be thought of as a field-free bound potential being tilted by a static electric field representing the laser at the peak value. Such a tilt in the potential will suppress the potential barrier on the one side and enlarge the probability of quantum tunnelling. If the peak field strength is high enough, the potential barrier can be suppressed so much that it is lower than the initial energy level. This picture is convenient in explaining the transition from multiphoton ionization to tunnelling ionization to over-the-barrier ionization (or barrier suppression ionization).

The over-the-barrier ionization picture is successful in the low laser frequency limit. It works well when the period of the electric field is much slower than the orbital periods of the relevant levels. In such a case, the field can be regarded as static and electrons can effectively escape the suppressed potential barrier.

In the opposite limit, when the optical period is much greater than the orbital period, the electron cannot escape before the lowered potential becomes a raised potential. Following such reasoning, the barrier suppression picture loses its validity in the high-frequency limit. A concrete example is illustrated in figure 7, in which the end-of-pulse ionization signal is plotted as a function of laser frequency. The figure shows that the degree of stabilization increases with increasing laser frequency. We note that the oscillation observed in figure 7 can be related to the oscillation in figure 5 .

\section{Conclusion}

We have re-examined photoionization from a zero-range potential as a function of field intensity, and under different pulse turn-on and turn-off conditions. The ionization found in weak fields is consistent with the perturbation theory prediction. For a high-frequency laser

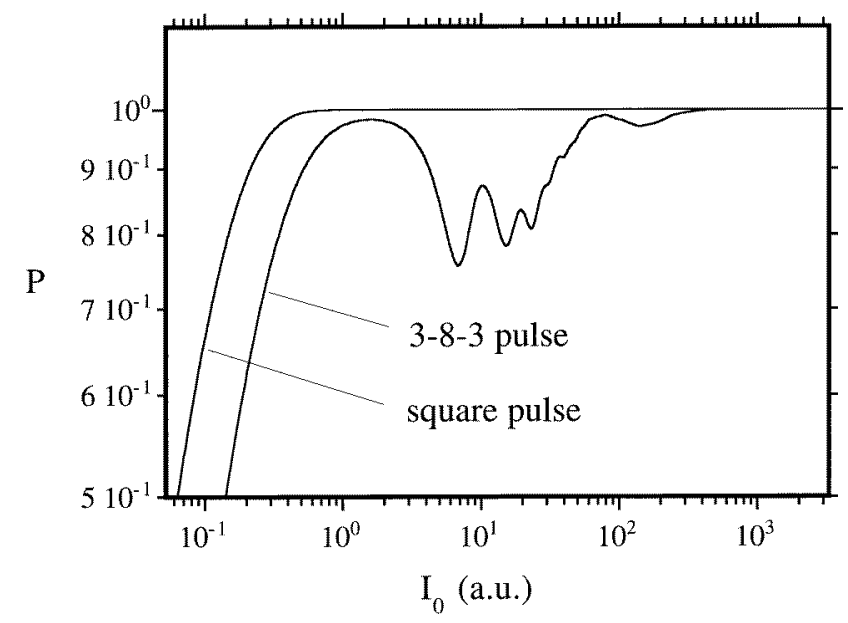

Figure 6. End-of-pulse ionization is plotted as a function of laser peak intensity. Results from two different laser pulse shapes are compared. The lower curve is replotted on a different scale from figure 5, but uses the same data shown there for 14-cycle pulses with a 3-8-3 cycle shape and sine-squared turn-on and turn-off. The upper curve is produced from 14-cycle laser pulses with a 'square', or abrupt turn-on, turn-off, shape. The laser frequency remains $\omega=1 \mathrm{au}$. It is evident that stabilization is absent from the square pulse ionization. 


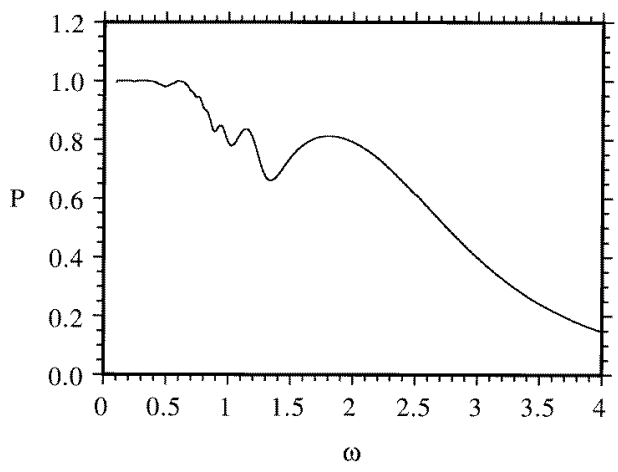

Figure 7. End-of-pulse ionization is plotted as a function of laser frequency, for pulses with the smooth 3-8-3 cycle shape of figures 4-6. The peak laser field strength $E_{0}=4$ au. An overall increase in the degree of stabilization is seen with the increase of laser frequency.

field we found clear evidence of dynamic stabilization in a wide range of laser intensities. This conclusion about stabilization is in disagreement with three previous investigations (Chen and Bernstein 1993, Krainov and Preobrazhenskii 1993, Geltman 1994, 1995), but we have presented evidence that a finite (although very rapid) turn-on is essential in stabilizing the atom. Statements based on instantaneous turn-on (Geltman 1994, 1995) must not be accepted generally. We would like to remark that the Chen and Bernstein calculation dealt with a one-dimensional electron moving in a bound potential with an impenetrable wall (Chen and Bernstein 1993). It is known that stabilization requires the distortion of the electron trajectory in the oscillating laser field so that its probability near the nucleus, which causes ionization, is small. It is not clear that the impenetrable wall potential of Chen and Bernstein permits such distortion of the electron trajectory. Therefore, we would note that the Chen and Bernstein result, often quoted (Geltman 1995) as supporting the case against the existence of stabilization, may be irrelevant to the discussion of stabilization. The stabilization observed here is in agreement with that found in another potential ( $\mathrm{Su}$ et al 1990, 1993, Su and Eberly 1990, 1991, Burnett et al 1993).

\section{Acknowledgments}

One of the authors (QS) thanks Dr P Agostini for bringing one of the references to his attention. The authors acknowledge helpful discussions with $\mathrm{M}$ Gavrila, R Grobe and K C Kulander, and a friendly correspondence with both A Fring and S Gettman. This research was supported by an award from the Research Corporation and by NSF grants PHY9511582 and PHY9631245. A URG award from Illinois State University is also acknowledged. Computing resources were supported by Illinois State University and the Pittsburgh Supercomputing Center.

\section{References}

Agostini P, Fabre F, Mainfray G, Petite G and Rahman N K 1979 Phys. Rev. Lett. 421127

Balcou P, L'Huillier and Escande D 1996 Phys. Rev. A 533456

Bucksbaum P H and Freeman R R 1991 J. Phys. B: At. Mol. Opt. Phys. 24325

Burnett K, Reed V C and Knight P L 1993 J. Phys. B: At. Mol. Opt. Phys. 26561

Chen Q, Bernstein I B 1993 Phys. Rev. A 474099 
Dörr M, Potvliege R M and Shakeshaft R 1990 Phys. Rev. Lett. 642003

1991 Phys. Rev. A 433729

Eberly J H, Javanainen J and Rzazewski K 1991 Phys. Rep. 204331

Eberly J H and Kulander K C 1993 Science 2621229

Feit M D, Fleck Jr J A and Steiger A 1982 J. Comput. Phys. 47412

Ferray M, L'Huillier A, Li X F, Lompre L A, Mainfray G and Manus C 1988 J. Phys. B: At. Mol. Opt. Phys. 21 L31

Figueira de Morisson Faria C, Fring A and Schrader R 1997 J. Phys. B: At. Mol. Opt. Phys. submitted

Fring A, Kostrykin V and Schrader R 1996 J. Phys. B: At. Mol. Opt. Phys. 29 in press

Gavrila M and Kaminski J Z 1984 Phys. Rev. Lett. 52613

Geltman S 1994 J. Phys. B: At. Mol. Opt. Phys. 271497 1995 Chem. Phys. Lett. 237286

Gersten J I and Mittleman M H 1976 J. Phys. B: At. Mol. Phys. 92561

Grobe R and Fedorov M V 1992 Phys. Rev. Lett. 682592

Grozdanov T P, Krstic P S and Mittleman M H 1990 Phys. Lett. A 149144

Haan S L 1996 Private communication

Henneberger W C 1968 Phys. Rev. Lett. 21838

Javanainen J, Eberly J H and Su Q 1988 Phys. Rev. A 383430

Krainov V P and Preobrazhenskii M A 1993 JETP 76559

Kramers H A 1956 Collected Scientific Papers (Amsterdam: North-Holland) p 272

Kulander K C 1987 Phys. Rev. A 35445

Kulander K C, Schafer K J and Krause J L 1991 Phys. Rev. Lett. 662601

Law C K, Su Q and Eberly J H 1991 Phys. Rev. A 447844

McPherson A, Gibson G, Jara H, Johann U, Luk T S, McIntyre I A, Boyer K and Rhodes C K 1987 J. Opt. Soc. Am. B 4595

Millack T 1993 J. Phys. B: At. Mol. Opt. Phys. 264777

Mostowski J and Eberly J H 1991 J. Opt. Soc. Am. B 81212

Pont M and Gavrila M 1990 Phys. Rev. Lett. 652362

Pont M, Walet N, Gavrila M and McCurdy C W 1988 Phys. Rev. Lett. 61939

Sanpera A, Su Q and Roso-Franco L 1993 Phys. Rev. A 472312

Su Q 1993 Laser Phys. 2241

1996 ICONO'95: Fundamentals of Laser-Matter Interaction (Proc. SPIE 2796) ed K N Drabovich and N I Koroteev, p 11

Su Q and Eberly J H 1990 J. Opt. Soc. Am. B 7564

1991 Phys. Rev. A 432474

Su Q, Eberly J H and Javanainen J 1990 Phys. Rev. Lett. 64862

Volkova E A, Popov A M and Smirnova O V 1994 JETP 79736

Yao G and Chu S-I 1992 Phys. Rev. A 456735 